\title{
X-RAY STRUCTURE PERFECTION DIAGNOSTICS OF SLIGHTLY DISTORTED SILICON CRYSTALS IN THE BRAGG CASE OF DIFFRACTION
}

\author{
V. KHRUPA* \\ Department of Physics, Science Laboratories, University of Durham \\ South Road, Durham, DH1 3LE, U.K. \\ S. Krasulya, V. Machulin, L. Datsenko \\ AND J. Auleytner \\ Institute of Physics, Polish Academy of Sciences \\ Al. Lotników 32/46, 02-668 Warsaw, Poland
}

Institute of Semiconductor Physics of the National Academy of Sciences, Kiev, Ukraine

\begin{abstract}
A new approach to structure perfection diagnostics of dislocation-free silicon crystals has been developed using the Bragg case of diffraction. The approach is being based on successive measurements of integral reflectivity and the spatial intensity distribution of reflected beam on the same diffraction planes of a real crystal by means of a single crystal diffractometer.
\end{abstract}

PACS numbers: $81.40 .-\mathrm{z}, 61.66 . \mathrm{Bi}$

\section{Introduction}

The Bragg case of the X-ray diffraction has been traditionally considered to be weakly sensitive to structure defects in the volume of a crystal. It was connected with the known fact that the diffraction maximum was formed near the crystal surface that means at a depth close to the value of the extinction length $\Lambda$.

However, the experimental investigations [1-5] carried out for the recent years have shown that the above-mentioned Bragg case of diffraction, under certain conditions, revealed rather weak structure distortions for which the level of the Debye-Waller static factor $L$ was ca. 0.999. For this purpose, first of all, the absorption length $t_{\mathrm{a}}\left(t_{\mathrm{a}}=1 / \mu_{0}\right.$, where $\mu_{0}$ is a leaner photoelectric absorption coefficient) must considerably exceed the extinction length $\Lambda$ what can be achieved by utilizing of hard X-ray radiation.

The aim of this paper is development of the method [3-5] for creation of the more simple and informative "single crystal" approach in structural diagnostics of weakly distorted $\left(L, 10^{-2}-10^{-3}\right)$ Si crystals.

\footnotetext{
*Permanent address: Institute of Semiconductor Physics, NAS, Kiev, Ukraine.
} 


\section{Theoretical background}

The integral reflectivity $R_{\mathrm{i}}$ of slightly distorted crystals $(L \ll 1$ and $\mu_{\mathrm{d}} \ll \mu_{0}$, where $\mu_{\mathrm{d}}$ is the coefficient of additional absorption due to diffuse scattering) consisting of the coherent $R_{\mathrm{B}}$ and diffuse $R_{\mathrm{D}}$ components can be written for the Bragg case of diffraction in the following way [6]:

$$
R_{\mathrm{i}}=R_{\mathrm{B}}+R_{\mathrm{D}}=\frac{16}{3 \pi} \frac{C Q \Lambda}{\gamma_{0}}+\frac{L Q}{\mu+\mu_{\mathrm{d}}},
$$

where $Q$ is the kinematical reflectivity of the unity length of an ideal mosaic crystal. $C$ and $\gamma_{0}$ stand for the polarization factor and the cosine of the Bragg angle $\theta_{\mathrm{B}}$, respectively. The first term in Eq. (1) is reflectivity $R_{\mathrm{i}}^{\mathrm{p}}$ for a perfect crystal. The diffuse component $R_{\mathrm{D}}$ can be particularly determined by the analysis of a spatial distribution (over coordinate $x$ counting from the point of radiation incidence on a crystal) of an intensity $I_{\mathrm{DS}}(x)$ registered by a detector with a narrow entrance slit $S$ for the relatively large $x(x>3 \Lambda)$ :

$$
I_{\mathrm{DS}}(x)=2 L Q \frac{I_{0} S}{\sin 2 \theta_{\mathrm{B}}} \exp \left[-\frac{\left(\mu_{0}+\mu_{\mathrm{d}}\right) x}{\cos \theta_{\mathrm{B}}}\right],
$$

$I_{0}$ is an intensity of the incident beam of X-rays which is connected in the case of the single crystal spectrometer with the measured integral intensity $I_{\mathrm{R}}$ of the Bragg diffraction maximum:

$$
I_{0}=I_{\mathrm{R}} / R_{\mathrm{i}}
$$

where $R_{\mathrm{i}}$ is described by the formula (1). The value of $\mu_{\mathrm{d}}$ parameter may be determined from the slope of the graph $\ln I_{\mathrm{DS}}(x)$. Hence, one can calculate the value of the Debye-Waller factor $L$ using the following relation:

$$
I_{\mathrm{R}} / I_{\mathrm{DS}}(x)=M\left(1+R_{\mathrm{B}} / R_{\mathrm{D}}\right) \approx M\left[\frac{\left(\mu+\mu_{\mathrm{d}}\right) R_{\mathrm{i}}^{\mathrm{P}}}{Q}\left(\frac{1}{L}-1\right)+1\right],
$$

where $M=\sin 2 \theta_{\mathrm{B}} / 2 S\left(\mu_{0}+\mu\right) \exp \left[-\left(\mu_{0}+\mu\right) x / \cos \theta_{\mathrm{B}}\right]$, which was obtained by means of (1), (2) and (3) formulae.

Thus, the essence of the proposed approach for determination both the $L$ and $\mu_{\mathrm{d}}$ parameters consists in successive measurements of the $R_{\mathrm{i}}$ value and the spatial distribution $I_{\mathrm{DS}}(x)$ for the same reflection. Contrary to Ref. [7] our method does not need any standard sample with a perfect structure.

\section{Preparation of samples and peculiarities of experiments}

Four silicon crystals were prepared from ingots grown by the Czochralski method. All of them have a high concentration of oxygen (up to $10^{18} \mathrm{~cm}^{-3}$ ). First three of them $(1,2,3)$ had the 111 orientation. The fourth sample was prepared for obtaining of reflections from the 100 plane. The sample 3 has a low density $N_{\mathrm{d}}$ of dislocation $\left(N_{\mathrm{d}}=5 \times 10^{3} \mathrm{~cm}^{-2}\right)$. The others were dislocation-free according to $\mathrm{X}$-rav topography. The samples 2 and 4 were annealed at $850^{\circ} \mathrm{C}$ during 2 and 4 hours, respectively, to create microdefects in a bulk $\left(\mathrm{SiO}_{2}\right.$ clusters or dislocation loops).

The measurements of the $I_{\mathrm{R}}$ of the 111, 333, 444, 555, 777 and $400 \mathrm{Bragg}$ reflections were carried out by means of the single crystal spectrometer using 


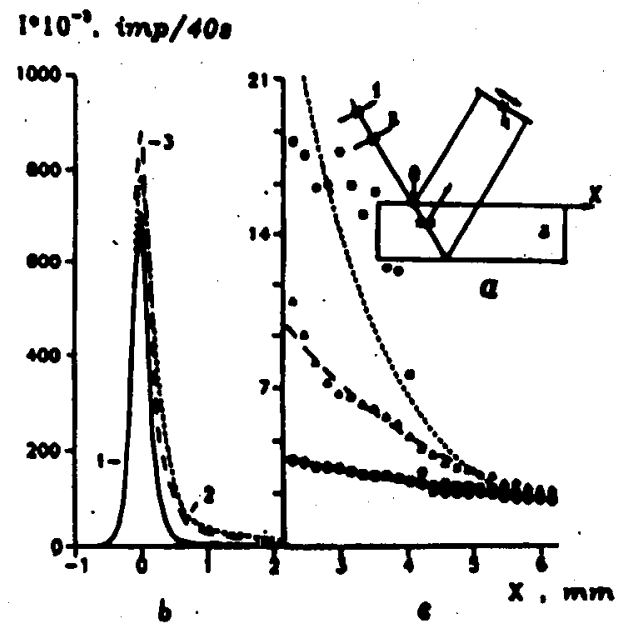

Fig. 1. The intensity spatial distribution profiles for the samples $1-0,2-\Delta$ and $3-\square$. The 444 Bragg reflection. (a) is a sketch of the experimental setup. 1, 2 collimating slits (the divergence of the beam is about $3^{\prime}$ ), 3 - the sample under investigations, 4 - the detector slit, $x$ - the coordinate measured from the point of incidence (0) along the crystal surface; (b) is the region near the diffraction maximum; (c) - the tails of the diffuse scattering function $I_{\mathrm{DS}}(x)$.

$\mathrm{Ag} K_{\alpha_{1}}$ radiation. The registration of the spatial distribution of intensities $I_{\mathrm{DS}}(x)$ of the reflected beams was performed by varying the position of the detector with a narrow entrance slit $(S=0.05 \mathrm{~mm})$ near the $2 \theta_{\mathrm{B}}$ angle (Fig. 1a). The dependence of the coordinate $x$ on the surface of a sample on the penetration depth $t[4,7]$ was determined by the formula $t=0.5 \times \tan \theta_{\mathrm{B}}$.

\section{Results and discussion}

The measured values of the $R_{\mathrm{i}}$ as well as meaning of the $R_{\mathrm{i}}^{\mathrm{p}}$ calculated by the dynamical theory of scattering for a perfect crystal are presented in Table together with the integral characteristics of crystal perfection $L$ and $\mu_{\mathrm{d}}$. The character of the spatial distribution of the intensity $I_{\mathrm{DS}}(x)$ of the 444-Bragg diffracted beam of the $\mathrm{Ag} K_{\alpha_{1}}$ radiation in the samples 1-3 and the functions $\ln I_{\mathrm{DS}}(x)$ for the sample 4 in its initial state and after annealing are shown in Fig. 1b, c and Fig. 2, respectively.

One can see from Fig. 1 the diffraction maximum and tails of diffuse scattering in the non-annealed sample 1 have the lowest level while comparing with these values for the annealed sample 2 and for the sample 3 containing dislocations. The same weak structure distortions as in the sample 1 took place probably in the initial state of the sample 4 (see the lower line in Fig. 2).

The deformation fields of microdefects situated at the depth of $\mu_{0}^{-1}$ gave noticeable diffuse scattering even in these relatively perfect $\left(L \cong 10^{-2}\right)$ crystals (see the Fig. 1b, c and Fig. 2). The experimental dependences $\ln I_{\mathrm{DS}}(x)$ for the sample 4 can be relatively well approximated by the straight lines (Fig. 2). It 
TABLE

The meanings of the integral characteristics of structure perfection and the diffraction parameters $R_{\mathrm{i}}, R_{\mathrm{D}} / R_{\mathrm{B}}$.

\begin{tabular}{|c|c|c|c|c|c|c|}
\hline Reflection & $\begin{array}{l}\text { Sample } \\
\text { number }\end{array}$ & $\mu_{\mathrm{d}}\left[\mathrm{cm}^{-1}\right]$ & $L \times 10^{3}$ & $R_{\mathrm{i}} \times 10^{7}$ & $R_{\mathrm{i}}^{\mathrm{p}} \times 10^{7}$ & $R_{\mathrm{D}} / R_{\mathrm{B}}$ \\
\hline \multirow{3}{*}{333} & 2 & 1.5 & 11 & 39.5 & \multirow{3}{*}{27.3} & 0.5 \\
\hline & & & & & & \\
\hline & 3 & 0.5 & 12 & 43.4 & & 0.6 \\
\hline \multirow{3}{*}{444} & 2 & 0.5 & 13 & 32.6 & \multirow{3}{*}{20.1} & 0.6 \\
\hline & & & & & & \\
\hline & 3 & 1.5 & 20 & 36.0 & & 0.8 \\
\hline \multirow{3}{*}{777} & 2 & 1.0 & 31 & 2.4 & \multirow{3}{*}{1.8} & 0.4 \\
\hline & & & & & & \\
\hline & 3 & 0.5 & 74 & 3.5 & & 1.0 \\
\hline 400 & 4 & 0.5 & 13 & 13.4 & 6.2 & \\
\hline
\end{tabular}

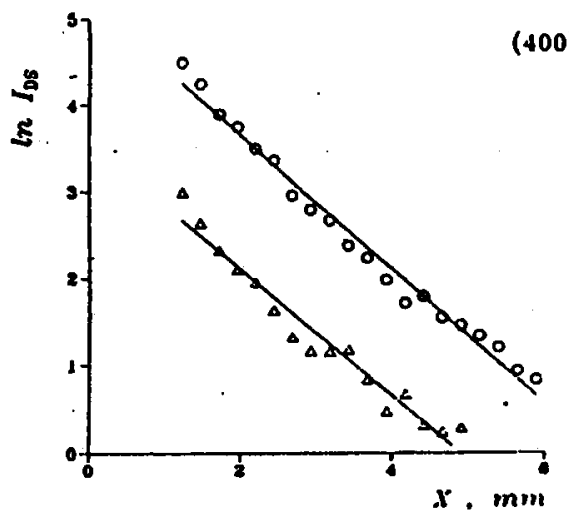

Fig. 2. Dependences $\ln I_{\mathrm{DS}}(x)$ for the 400 Bragg reflection in the initial state of the sample $4-\Delta$ and that after annealing $\left(850^{\circ} \mathrm{C}, 4 \mathrm{~h}\right)-\mathrm{o}$.

testifies to the homogeneous distribution of microdefects in the volume of the crystal.

The $\mu_{\mathrm{d}}$ values obtained from the graphs $\ln I_{\mathrm{DS}}(x)$ are relatively small comparing with the $\mu_{0}$. It testifies to weak influence of the parameter $\mu_{\mathrm{d}}$ on the experimentally measured values of the Bragg diffracted beam intensities. One can see from Table that the level of the Debye-Waller factor grows by transition to reflections of a higher order what is in agreement with the theory [8]. The level of diffuse scattering (see the ratio $R_{\mathrm{D}} / R_{\mathrm{B}}$ ) grows in more distorted samples. The "velocity" of the $L$ increasing with growing of the order $N$ of reflection is higher for 
the sample with dislocations $[8,9]$. This fact is connected with a different character of the $L(N)$ dependences for dislocations and that for microdefects [8, 9].

All of $R_{\mathrm{i}}$ values in the studied samples turned out to be higher than the corresponding ones for a perfect crystal. This proves a sufficient sensitivity of our measurements for weak structure distortions in near perfect crystals.

Mean values of the radii $r$ and the concentration $n$ of microdefects calculated according to [8,9] from the integral characteristics $L$ and $\mu_{\mathrm{d}}$ for the annealed crystal 4, i.e. $r=0.2 \mu \mathrm{m}, n=8 \times 10^{7} \mathrm{~cm}^{-3}(\Delta r / r, \Delta n / n \cong 15 \%)$ are close to corresponding parameters obtained by means of the independent diffractometrical measurements carried out by means of the two wavelength methods (see our publication in this issue).

The proposed approach to structure perfection diagnostics of slightly distorted crystals basing on the successive measurements of both the reflectivity and the intensity spatial distribution of the Bragg diffracted beams on the same sample containing structural defects permits to expand essentially the possibilities of the single crystal diffractometer [10] especially applying synchrotron radiation. This approach may be a useful supplement for more complex in practical realization methods of structure diagnostics such as high energy synchrotron diffraction, small angle scattering, neutron backscattering and so on.

Financial support from the Royal Society (Great Britain), Ukrainian Scientific and Technology Committee as well the Committee for Scientific Research (Poland) is acknowledged.

\section{References}

[1] P.I. Klimanek, K.H. Hanish, Cryst. Res. Technol. 18, 361 (1983).

[2] A.N. Ivanov, P.I. Klimanek, Ya.A. Skakov, Krystallografia 28, 109 (1993).

[3] V.I. Khrupa, Metallofizika 13, 49 (1991).

[4] I.R. Entin, V.I. Khrupa, J. Appl. Cryst. 24, 403 (1983).

[5] V.I. Khrupa, D.O. Grigoriev, M.Ya. Skorokhod, L.I. Datsenko, Acta Phys. Pol. A 86, 591 (1994).

[6] V.G. Bar'yakhtar, V.V. Nemoshkalenko, V.B. Molodkin, S.I. Olikhovskii, E.N. Gavrilova, E.N. Kislovskii, V.V. Kochelab, A.I. Nizkova, Metallofizika 15, 18 (1993).

[7] V.I. Khrupa, I.R. Entin, Metallofizika 13, 117 (1991).

[8] L.I. Datsenko, V.B. Molodkin, M.E. Osinovskii, Dynamical Scattering of X-rays by Real Crystals, Naukova Dumka, Kiev 1988 (in Russian).

[9] V.F. Machulin, V.I. Khrupa, X-ray Structure Perfection Diagnostics of Weakly Distorted Crystals, Naukova Dumka, Kiev 1995 (in Russian).

[10] L.I. Datsenko, A.N. Gureev, V.I. Khrupa, E.N. Kislovskii, V.P. Kladko, A.I. Nizkova, I.V. Prokopenko, M.Ya. Skorokhod, Author certificate N1255906 of the USSR, Otkrytiya and Izobreteniya 33 (1986). 